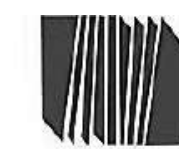

UNIVLRSIDADPLDAGOGICA EXPER HAENTAL LIB CRTROOR

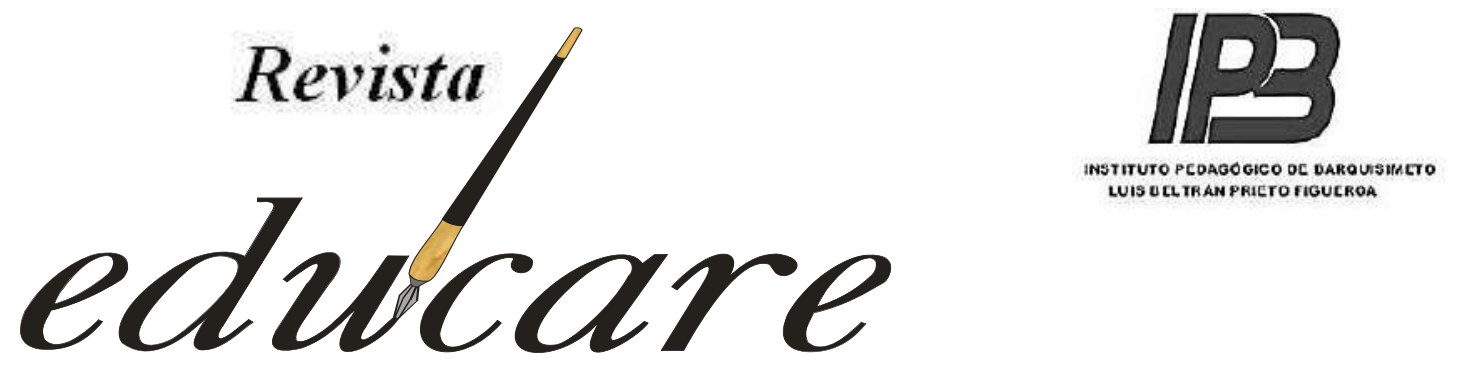

Órgano Divulgativo de la Subdirección de Investigación y Postgrado del Instituto Pedagógico de Barquisimeto "Luis Beltrán Prieto

Figueroa"

BARQUISIUEIO - EDO. LARA - VEAEZUEA

\title{
DIDÁCTICA CRÍTICA EN LA ENSEÑANZA DE LA MATEMÁTICA DESDE UNA VISIÓN ÉTICA
}

\author{
CRITICAL DIDACTICS IN TEACHING
}

MATHEMATICS FROM AN ETHICAL VISION

RODOLFO ANTONIO BASTARDO LUNAR*

UNIVERSIDAD POLITÉCNICA TERRITORIAL DEL OESTE DE SUCRE “CLODOSBALDO RUSSIÁN" 


\begin{tabular}{|c|c|}
\hline$\underline{E N S A Y O}$ & $\begin{array}{r}\text { Rodolfo A Bastardo L* } \\
\text { UPTOS }\end{array}$ \\
\hline Recibido: 27-01-2016 & Aceptado: $26-05-2016$ \\
\hline RESUMEN & ABSTRACT \\
\hline $\begin{array}{l}\text { El presente ensayo tiene como finalidad que el } \\
\text { docente adopte la didáctica crítica en la } \\
\text { enseñanza de la matemática desde una visión } \\
\text { ética, ya que actualmente el proceso de enseñanza } \\
\text { no está desarrollando en el discente un } \\
\text { pensamiento crítico que propicie la } \\
\text { transformación de los contenidos acorde con sus } \\
\text { necesidades. Debido a esto, se debe pensar en una } \\
\text { "didáctica distintiva" que permita crear un } \\
\text { vínculo afectuoso, partiendo de la ética y la } \\
\text { estética, logrando un cuestionamiento a los } \\
\text { principios de la escuela tradicional y tecnocrática, } \\
\text { impulsando una ruptura epistemológica que } \\
\text { vislumbre nuevos senderos en la enseñanza, que } \\
\text { parta de la historia actual del individuo. A manera } \\
\text { de conclusión, la didáctica crítica en la enseñanza } \\
\text { de la matemática destaca la necesidad de } \\
\text { sensibilizar a los docentes para que generen } \\
\text { acciones educativas con bases teóricas hacia una } \\
\text { pedagogía centrada en la moralidad, con carácter } \\
\text { ético y liberador. }\end{array}$ & $\begin{array}{l}\text { The purpose of this essay is for the teacher to } \\
\text { adopt critical didactics in the teaching of } \\
\text { mathematics from an ethical perspective, since } \\
\text { the teaching process is not currently developing } \\
\text { in the student a critical thinking that facilitates } \\
\text { the transformation of contents according to their } \\
\text { needs. Due to this, we must think of a } \\
\text { "distinctive didactics" that allow us to create an } \\
\text { emotional link, starting from ethics and } \\
\text { aesthetics, achieving a challenge to the } \\
\text { principles of the traditional and technocratic } \\
\text { school, promoting an epistemological rupture } \\
\text { that envisages new paths in the teaching, that } \\
\text { starts from the current history of the individual. } \\
\text { As a conclusion, the critical didactics in the } \\
\text { teaching of mathematics highlights the need to } \\
\text { sensitize teachers to generate educational } \\
\text { actions with theoretical bases towards a } \\
\text { pedagogy centered on morality, with ethical } \\
\text { character and releasing. }\end{array}$ \\
\hline $\begin{array}{l}\text { Descriptores: Didáctica crítica, enseñanza de la } \\
\text { matemática, ética. }\end{array}$ & $\begin{array}{l}\text { Keywords: Critical didactics, teaching of } \\
\text { mathematics, ethics. }\end{array}$ \\
\hline
\end{tabular}

\footnotetext{
* Actualmente cursante del Doctorado en Ciencias de la Educación ULAC. Licenciado en Matemáticas. Magister en Docencia de Educación Superior. Magister en Matemáticas. Profesor de Matemáticas en la Universidad Politécnica Territorial del Oeste de Sucre “Clodosbaldo Russian”. Venezuela, Cumaná Estado Sucre. Email: bastardolunar1@yahoo.es
} 


\section{INTRODUCCIÓN}

Con la didáctica crítica en la enseñanza de la matemática desde una visión ética, se pretende atender escenarios que exigen del docente un elevado nivel de comprensión, sensibilidad y compromiso social, y dejar atrás las prácticas educativas tradicionales basadas en la memorización como lo ha asegurado Álvarez (2006), al afirmar que "hay un predominio de la memorización y la repetición como estrategia de estudio y el docente mayoritariamente utiliza el monólogo, el dictado y los símbolos en el desarrollo de sus clases" (p.4). Asimismo ha prevalecido un dominio fuerte del racionalismo científico técnico, además una sobre-autoridad y saber por parte del docente.

Con base en esto, Freire (1970) describe este tipo de educación con los siguientes principios:

La educación bancaria mantiene y estimula la contradicción. De ahí que ocurra en ella que: A) el educador es siempre quien educa; el educando el que es educado. B) el educador es quien sabe; los educandos quienes no saben. C) el educador es quien piensa, el sujeto del proceso; los educandos son los objetos pensados. D) el educador es quien habla; los educandos quienes escuchan dócilmente (p.33).

Esta educación bancaria de la que nos habla Freire (1970) ha conllevado a prevalecer lo objetivo, lo medible y lo cuantificable en la enseñanza de la matemática en Latinoamérica, originando pasividad por parte del alumno y dejando a un lado la ética, la comunicación, la subjetividad y la intersubjetividad, entre otros.

$\mathrm{Al}$ respecto Alsina (2007) también agrega que:

Realizar una reflexión sobre la realidad como referente para nuestra actuación docente, prestando especial atención a las falsas realidades tan presentes aún en nuestra enseñanza e indicando las características deseables del realismo educativo. Gran parte del tiempo dedicado a la enseñanza de la matemática se dedica a la resolución de ejercicios rutinarios alejados de la vida cotidiana (p.85).

Con estas afirmaciones de Álvarez (2006), Freire (1970) y Alsina (2007), se podría pensar que la enseñanza de la matemática en Venezuela no está cumpliendo con las expectativas de desarrollar en el discente un pensamiento crítico y liberador que le permita transformar su realidad y conectar los contenidos en matemáticas con sus necesidades reales.

De acuerdo con los autores antes mencionados, la didáctica critica en la enseñanza de 
la matemática, desde una visión ética, tanto la memorización, como la educación bancaria y los ejercicios en matemática alejado de la cotidianidad le resulta inútil y deben ir quedando atrás, porque hoy en día existen nuevas teorías, nuevas posturas, nuevas oportunidades para crear e inventar, y nuevos enfoques que nos permiten dejar a un lado lo estático y lo irreflexivo.

Asimismo, lo afirmado por estos autores ha generado desconcierto, poco tiempo al estudio en el área de la matemática, sin embargo, por medio de este ensayo se busca que el docente, con una didáctica critica en la enseñanza de la matemática desde una visión ética, por medio del trabajo grupal y la investigación, guie a los estudiantes a una participación autónoma, creativa y al sentido crítico para que asimilen los conocimientos, los interiorice y los utilice, conduciéndolos a ser parte de los cambios que demanda la sociedad actual.

Así, en el contexto actual de la educación latinoamericana, en todos sus niveles, el proceso de enseñanza de la matemática no está cumpliendo con las expectativas de desarrollar en el alumno un pensamiento crítico y liberador, para transformar su realidad como lo ha planteado Tunnermann (2000), al hacer referencia que las instituciones educativas deben estructurarse no solo como transmisoras y difusoras de conocimientos, sino también como centro de pensamiento crítico. En ese mismo sentido, Freire (1976) argumenta que la educación verdadera es praxis, reflexión y acción del hombre sobre el mundo para transformarlo

En referencia a lo expresado por Tunnermann (2000) y Freire (1976), se debe pensar en una didáctica de la matemática más allá de la explicación como lo ha planteado Ranciere (2003) en su obra El Maestro ignorante, al exponer que hay que librarse del yugo del maestro explicador y por medio de esta categoría se hace necesaria la transformación de la educación venezolana en todos sus niveles en el área de la matemática, pero no solo en un orden técnico, sino también en un orden cultural y ético.

En otro orden de ideas, no se puede dejar de mencionar que el paradigma racionalista de la modernidad nos ha sumergido en una profunda crisis y por tanto, es preciso su cuestionamiento a la crítica, a la racionalidad moderna. En este sentido, Carmona (2007) señala que "a través del proceso de racionalización llevado a cabo por la modernidad la razón emancipadora se ha escindido en la razón científico - técnica, convertida en razón instrumental que coloniza y domina el mundo material e individual” (p.137). 
Lo expresado anteriormente coincide con lo que hemos dicho al inicio con respecto al fuerte dominio de la racionalidad técnica instrumental y de poder por parte del docente en la que sigue atrapada la enseñanza de la matemática en Latinoamérica y muy particularmente en Venezuela. Aquel sueño en el poder de la razón de organizar un mundo humano pleno de libertad y progreso no se ha realizado, por el contrario, lo que se constata es un mundo deshumanizado y los ideales o los grandes relatos modernos: el proyecto emancipador, el progresista, el innovador y el democrático han quedado en promesas y utopías.

Estos planteamientos nos obligan a reflexionar y tratar de comprender que la praxis educativa en la matemática en Venezuela sigue centrada en clases expositivas por parte del docente sin tomar en cuenta las capacidades ni las necesidades reales de los discentes y por ello, en la actualidad este conocimiento es percibido por los discentes de manera poco significativa, al sentir un desfase entre los contenidos y lo que son sus necesidades en cuanto a desarrollar en ellos sus talentos, creatividades y habilidades, para relacionarlos con otras especialidades como la ingeniería, la medicina, la sociología, la psicología, entre otras.

En este mismo orden, para Almeida (2002).

Los problemas que se presentan en el aprendizaje de la matemática son de diversa naturaleza, entre ellos están: rechazo o predisposición a su estudio, bajo rendimiento, deserción de las carreras, por ejemplo desatención de importantes aspectos conceptuales para dedicarse en exclusiva a los mecanismos de los procedimientos (p. 40).

Estas afirmaciones de Almeida (2002) concuerdan con las de Álvarez (2006), y para nadie es un secreto que el aprendizaje de la matemática por su diversidad en cuanto a su naturaleza ha penetrado en casi todos los ámbitos de la sociedad y es necesario revertir las consecuencias de estas aseveraciones, en vista de que todos los ciudadanos requieren saber algo de matemática para desenvolverse en la sociedad competitiva y globalizada en la que vivimos, sin embargo, a pesar de los adelantos científicos y tecnológicos del hombre en la actualidad, aún continúan los problemas en la enseñanza de la matemática en Venezuela.

Por otra lado, si los docentes se hacen los ciegos ante lo que acontece a su alrededor, sin hacer nada al respecto, se pierde la esencia de la ética. Por eso, no deben quedarse en el 
pasado, sino buscar propiciarles las herramientas a los estudiantes para que desarrollen sus propios pensamientos críticos reflexivos ante su entorno social, para que valoren sus actos desde su cotidianidad, por medio del trabajo grupal y la investigación, pues solo así se estará educando para la vida, con una verdadera ética que parta desde la historia actual del individuo.

\section{LA DIDÁCTICA CRÍTICA EN LA EDUCACIÓN}

Según Anónimo, (s.f.), afirma que:

La didáctica crítica surge hacia la mitad del siglo XX como un cuestionamiento a los principios de la escuela tradicional y la didáctica tecnocrática. Se manifiesta por la reflexión colectiva entre docentes y discentes sobre problemas que se encuentran en su propio contexto (p.4).

En este mismo orden de ideas, "Observa como principal problema de la educación la cuestión política más que la técnica, reúne elementos del psicoanálisis y explica a partir de las interacciones sociales, el proceso de enseñanza en la educación” (ibíd. p.5). Con esta categoría en la escuela crítica es necesaria una renovación de la enseñanza, una evaluación constante de ésta y un diálogo consciente.

"En esta corriente es importante el análisis institucional de la escuela que se rige por normas que intervienen en la relación pedagógica del docente con sus alumnos. Bajo esta lógica, el profesor solo puede relacionarse institucionalmente con sus discentes” (ibíd. p.5). "La puesta en marcha de la didáctica crítica se basa en estrategias de reflexión, de discusiones y negociación constante que facilitan a los estudiantes acceder a un escenario donde ellos juegan un papel protagónico, al igual que los profesores” (ibíd. p.4).

En efecto, abordaremos la didáctica crítica desde diversas concepciones:

Para Rodríguez (1997) "la didáctica crítica la entiende como una ciencia teóricopráctico que orienta la acción formativa, en un contexto de enseñanza y aprendizaje mediante procesos tendencialmente simétricos de comunicación social, desde el horizonte de una racionalidad emancipadora" (p.40). Con estas apreciaciones de la didáctica critica en la educación, se le permitirá al alumno participar en su proceso formativo tomando en cuenta su libertad para resolver problemas, también transforma el papel del docente y del 
alumno, es decir, se busca aprender el uno del otro, y ver la comunicación como medio para la generación de conocimiento en constante interacción con el entorno.

Por otro lado, para Morán (1996) "la didáctica critica es todavía una propuesta en construcción, que se va configurando sobre la marcha; una tendencia educativa que no tiene un grado de caracterización como es el caso de la didáctica tradicional y la tecnología educativa" (p.180); en esta conceptualización de la didáctica critica se busca analizar la práctica educativa en donde el aprendizaje sea concebido como un proceso que manifieste constantes momentos de ruptura y reconstrucción, buscando diferenciarse de los planteamientos mecanicistas del aprendizaje, como han sido enfocados desde la didáctica tradicional, la cual se ha centrado más en el resultado que en el proceso.

Con la didáctica crítica se busca romper el vínculo dependiente del docente, el alumno y la materia objeto del conocimiento, con la idea de reconstruir el conocimiento a partir de la reflexión colectiva y la problematización como lo ha planteado Freire (1970) en su obra Pedagogía del oprimido.

De estas dos conceptualizaciones el primero la concibe como una ciencia teóricapráctica orientadora de la formación del alumno y el segundo como una categoría en construcción que se va configurando sobre la marcha. En ambas apreciaciones se nota un despertar implícito de la conciencia y la autonomía personal, en función de descubrir el contexto y las teorías que la atraviesan, purificando las distorsiones subyacentes en su aplicación y mejorarla con miras a la emancipación de los individuos y de la sociedad.

Se plantea no ver con esta categoría al docente como un técnico, para mejorar los procedimientos encaminados a procurar un mayor rendimiento académico tal como se ha venido dando con la didáctica tradicional y la tecnología educativa en la actualidad.

En otras palabras, cabe destacar que por medio de la didáctica crítica en la educación, el docente debe propiciar la autoconciencia del aprendizaje por ser considerado un proceso inacabado. Asimismo se pretende formar alumnos críticos, pensantes y creativos mediante el desarrollo de habilidades superiores del pensamiento. Esta categoría, en el proceso de enseñanza de la matemática desde una visión ética, permitirá influir para que los alumnos investiguen, opinen, cuestionen, apliquen el conocimiento, dentro de su contexto social, cultural o educativo, además aprender investigando, puede ser una buena metodología de la didáctica crítica y no ver los contenidos como desconectados unos de otros como productos 
impersonales, ahistóricos y descontextualizados.

Es decir, el docente debe relacionar su práctica educativa con la teoría, la investigación y la práctica bajo una perspectiva indisoluble, desde el aula. Lo que significa que con esta categoría se debe analizar el contexto, la institución y el aula con el propósito de generar elementos teóricos de la didáctica critica coherentes y que propicien el desarrollo integral del propio docente, de sus colegas y de sus alumnos, desde la ética.

\section{La Didáctica Crítica En La Enseñanza De La Matemática}

Con la didáctica crítica en la enseñanza de la matemática desde una visión ética pretendemos, como lo asegura Goñi et al. (2000), que el reto es "lograr que la matemática no sea algo externo al alumno, a su experiencia vital (...) que logremos plantearles problemas que puedan integrarlos en un contexto próximo a ellos, que les permitan desarrollar capacidades realmente útiles fuera de la vida académica" (p.94). Estos aportes buscan humanizar esta ciencia y debatir nuevos enfoques y aportes teóricos en cuanto a su enseñanza ya que, en la actualidad seguimos encontrándonos con clase de matemática donde los docentes siguen dejando a un lado la discusión de ideas, la comunicación de pensamientos entre éstos y los discentes, de allí la necesidad de incorporar la didáctica crítica en la enseñanza de la matemática desde una visión ética en Latinoamérica.

En consecuencia, al percibir su enseñanza y aprendizaje con métodos tradicionales, basados solo en abstracciones y sin favorecer la concreción del mismo, produce un aprendizaje descontextualizado e irrelevante para el alumno. Con base en esto es conveniente considerar lo afirmado por Confrey (1991) que "el aprendizaje es una actividad interactiva, tanto individual como construida. En la enseñanza y el aprendizaje de la matemática profesores y alumnos construyen matemáticamente interpretaciones y promueven la comprensión de su significado matemático" (p.35).

Estas afirmaciones de Confrey (1991) pueden ayudar al considerar la didáctica como elemento emancipador, la ciencia de la didáctica trata de cambiar al estudiante como persona individual, sus actitudes, valores, juicios, sentimientos, capacidades, aptitudes y mentalidades, y es aquí donde la ética parte desde la concepción del hombre como persona, enfocándose principalmente en el comportamiento, que no es más que otra cosa que la moralidad. Cada individuo tiene un modo de ser singular, con una forma de actuar propia, o 
sea, es responsable de sus actos. Sin embargo estos actos van a estar muy relacionados con la percepción que ese individuo tiene de lo que para él es lógico o bueno.

Por otro lado, Uzuriaga, Vivian y Martínez (2006), afirman que:

La educación matemática debe ser valorada y rescatada por los matemáticos pues es claro que combinar una muy buena solidez y conocimientos matemáticos con las teorías pedagógicas y centrar nuestra atención en desarrollar, o por lo menos usar adecuada y críticamente, metodologías que le permitan a nuestros alumnos un aprendizaje a lo largo de la vida, a aprender a aprender, aprender a emprender, aprender a ser, aprender a conocer, aprender a trabajar en colaboración, a valorar el contexto histórico cultural (p. 269).

Considerando este planteamiento de Uzuriaga y otros (2006) induce a reflexionar la didáctica crítica en la enseñanza de la matemática desde una visión ética, en otras palabras, se apuesta por una nueva manera de asumir la enseñanza de la matemática, en los escenarios académicos actuales. Esto significa evaluar y cuestionar algunos modos de enseñanza, a fin de ubicar al docente, al alumno y a las áreas de aprendizaje en una nueva dimensión ontológica, epistemológica y metodológica.

En tal sentido, se propone un proceso de enseñanza de la matemática basado en las relaciones humanas, donde se le dé mayor importancia al desarrollo del ser como ente experiencial, dándole a la acción comunicativa una nueva visión de incorporar bases de integración social, ya que a través de las vivencias cotidianas de los entes involucrados en el proceso de enseñanza y aprendizaje se pueda apuntar hacía una nueva enseñanza más humanizadora del ser, donde se le de relevancia a la ética y a la estética. Permitiendo visualizar las potencialidades de los estudiantes, para mantener sentimientos de amor durante la práctica pedagógica, ya que la idea está en dejar que se desarrollen las emociones, creaciones y los sueños de los educandos.

Continuando con esta realidad, Cortina (2012) explica sobre la ciudadanía planetaria, donde la moralidad del ser humano se acompaña con las relaciones sociales, para lo cual se debe dejar a un lado el individualismo y el ser sumiso generados por el poder económico arrasador, como lo es la globalización.

Cabe agregar que autores como Savater (1997) en su obra El valor de educar, Levinas (2002) en su obra Totalidad e infinito y Jean-Luc (2000) en su obra La comunidad inoperante, hacen un llamado a considerar lo humano y ver que las deficiencias de uno sean 
las nuestras y considerar al otro como al nosotros dejando a un lado el individualismo y partiendo de estas premisas hago un llamado a incorporar la didáctica critica en la enseñanza de la matemática desde una visión ética, tomando en cuenta las consideraciones de dichos autores en sus respectivas obras.

La coincidencia de estos tres autores está en darle cabida a la alteridad que no es más que reconocer al otro y este será un elemento clave en este ensayo. También coinciden en ver la libertad del otro como nuestra propia libertad. La diferencia de estos autores, está en que Savater (1997) hace hincapié en esta obra en lo humano como un deber y lo importante de llegar a serlo, ya que no basta con nacer humano y lo relaciona con la solidaridad y benevolencia entre hermanos y además ver la libertad como una liberación de la ignorancia. Levinas (2002) nos hace ver en esta obra que es a través del lenguaje, ya que éste crea la relación sujeto-objeto. Y Jean-Luc (2000) nos plantea en esta obra una inclinación del uno por el otro, pero en comunidad.

Estas obras han sido de gran relevancia para el desarrollo de éste ensayo, primero nos invita a sembrar lo humano como una semilla que debemos germinar, en función de la solidaridad y el buen trato hacia nuestros semejantes independientemente de su condición física, económica, social, de raza, de credo o creencia, entre otros. También sembrar el ideal que el estudiante supere al maestro, y la importancia de educar para que nuestros discentes sean cada día mejores ciudadanos. El llamado es a desarrollar el espíritu investigativo en docentes, estudiantes y propiciar el aprendizaje grupal, cultivando principios, valores, justicia y equidad.

En muchos contextos la didáctica critica ha dado sus resultados de acuerdo a estudios muy recientes y por ello el autor de este articulo aspira que con esta categoría en la enseñanza de la matemática en Latinoamérica y particularmente en Venezuela con las posturas de autores como: Rodríguez (1997), Morán (1996), Freire (1970), Savater (1997), Levinas (2002) y Jean-Luc (2000), que la han enfocado en sus obras como participativa, critica, dialéctica, relacional y sistémica que favorece el debate, la reflexión, la discusión en pequeños grupos y se fundamenta en la comunicación, el consenso, la seguridad, la convergencia, la unidad a través de valores compartidos, cooperativos, emancipadores, solidarios e intersubjetivos, se pueda fomentar la didáctica critica en la enseñanza de la matemática desde una visión ética. 
En este mismo sentido, la estética en esta tarea de educar, está en mantener sentimientos de amor durante el proceso educativo y en no quitarle las emociones, creaciones y los sueños de los educandos. La estética debe ir de la mano con la ética. El educador con ética visualiza individualmente las potencialidades y creatividades de sus discentes, porque la idea radica en no quitarles a nuestros alumnos sus sueños, sus esperanzas ni sus maneras de ver y enfrentar las cosas.

Asimismo, Freire (1966) también señala que "enseñar no existe sin aprender y viceversa" (p. 25). Efectivamente, como seres sociales, tenemos que romper con el modelo positivista técnico- instrumental, emanado de la modernidad.

También Lyotard, (citado en Peleteiro, 2006), afirma que "el proyecto modernista ha terminado, reina el azar y la incertidumbre ante las cuales la interacción y el dialogo se perfilan como los únicos capaces de arrojar una luz" (p. 54). Es por ello que en esta etapa que llamaríamos la postmodernidad se están propiciando la emergencia de nuevas maneras de pensar la realidad, de relacionarse, de aprender y de enseñar, de concebir el trabajo y el ocio, y esto generaría nuevas demandas en la educación latinoamericana, dejando atrás esa forma de enseñar que se ha venido aplicando antes de la aparición de las tecnologías de información en el proceso de enseñanza y aprendizaje de la matemática.

\section{CONSIDERACIONES FINALES}

Con la didáctica crítica se manifiestan los valores compartidos, cooperativos, emancipadores, solidarios e intersubjetivos, la relación entre la teoría y la práctica es indisociable, y el aprendizaje se concibe como un proceso grupal y en construcción que se define por los cambios individuales y sociales que se están dando a inicios de este siglo XXI. Con la inclusión de la didáctica crítica en la enseñanza de la matemática desde una visión ética se destaca la necesidad de sensibilizar a los docentes para que generen acciones educativas que permitan sentar las bases teóricas hacia una pedagogía centrada en la moralidad y ver al conocimiento como una construcción social con un carácter liberador y ético.

Además, la enseñanza es una actividad compleja, responsable y de mucho agrado y satisfacción, cuando está presente la vocación y acompañado de esto, obviamente la 
dedicación. Debemos ser profesionales reflexivos, profesionales críticos con nuestra práctica, como manera de ir mejorando cada día el quehacer diario en el aula de clases. Es por ello que la profesión docente ha sido una de las actividades que ha motivado al estudio y a la reflexión en la historia del pensamiento humano.

En estos tiempos de "crisis en la educación", se requieren docentes apasionado por lo que enseñan y saben mediante su ejemplo consoliden los principios de la ética profesional, y la ausencia de valores, muy de moda en la sociedad actual. Siempre enfocarnos en esa relación de lo que enseñamos y de lo que el alumno aprende con lo que será su quehacer diario como profesional. Cada día las reflexiones como facilitadores en el proceso de enseñanza y aprendizaje, la meta inicial tiene que ser lograr la atención del alumno, y esto se lograra a medida que el enfoque en el aula sea novedoso, distinto, personalizado.

Para concluir, el llamado es a las universidades, para que contribuyan con la formación de personal de alto nivel y fomenten el desarrollo científico y tecnológico, para emprender un proceso de transformación, que nos prepare para estos ambientes de cambio, que se respiran en esta segunda década de este siglo XXI, y es un gran reto que tiene una estrecha relación con la ética, ya que como seres sociales dependemos los unos de los otros.

\section{REFERENCIAS}

Anónimo. (s.f.). Didáctica. [Documento en línea].Universidad Interamericana para el Desarrollo.Disponible:http://moodle2.unid.edu.mx/dts_cursos_mdl/lic/ED/DC/S03/D C03_Lectura.pdf [Consulta: 2015, marzo, 25]

Almeida, P. (2002). Educación lúdica. Técnicas y juegos pedagógicos. Bogotá: D.CColombia: San Pablo.

Alsina, C. (2007). Si Enrique VIII tuvo 6 Esposas, ¿Cuántas tuvo Enrique IV? El Realismo En Educación Matemática y sus Implicaciones Docentes. Revista Iberoamericana de Educación. 43, 85-101.

Álvarez, Y. (2006). ¡Auxilio! no puedo con la matemática. Revista Iberoamericana de Educación Matemática. Equisángulo. 2(1), 4-16.

Carmona, M. (2007). La Educación y la crisis de la Modernidad. Hacía una Educación Humanizadora. [Revista en línea]. Revista de Artes y Humanidades UNICA, vol. 8, 
núm. 19, mayo-agosto, 2007, pp. 134-157 Universidad Católica Cecilio Acosta

Maracaibo: Venezuela. Disponible: http://www.redalyc.org:9081/articulo.oa?id=170 $\underline{118451008}$ [Consulta: 2015, abril 3]

Confrey, J. (1991). Learning to listen: A student's understanding of power of ten. In E von Glasersfeld (ed), Radical constructivism in mathematics education (pp. 111-138). Dondrecht, The Netherlands: Kluwer Academic-Publishers.

Cortina, A. (2012, Julio). Ética en tiempos de crisis [Periódico en línea]. El País. Ediciones el País S.L. Disponible: http://elpais.com/elpais/2012/06/13/opinion/1339587064_1 62579.html [Consulta: 2015, Marzo 31]

Freire, P. (1966). Pedagogía de la Autonomía: Saberes necesarios para la práctica educativa. [Libro en línea]. Sao Paulo, Brasil: Paz e Terra. Disponible: https://books.google.co.ve/books?id=OYK4bZG6hxkC\&printsec=frontcover\&dq=isb $\underline{\mathrm{n}: 9682320690 \# \mathrm{v}=\text { onepage \&q\&f}=\text { false }}$

Freire, P. (1970). Pedagogía del oprimido. Montevideo, Uruguay: Tierra Nueva.

Freire, P (1976). La educación como práctica de la libertad. México: Siglo XXI.

Goñi, J.; Alsina, C.; Ávila, D.; Burgués, C.; Comellas, J.; Corbalán, F.; García, M.; Hahn, C.; Serra, J. (2000). El curriculum de matemáticas en los inicios del siglo XXI. Barcelona, España: Graó.

Jean-Luc, N. (2000). La comunidad inoperante. [Documento en línea]. Santiago de Chile, Chile. Disponible: http://www.lacomunitatinconfessable.com/wp-content/uploads/ 2009/10/18223929-la-comunidad-inoperante-jeanluc-nancy.pdf [Consulta: 2015, Abril 15]

Levinas, E. (2002). Totalidad e infinito. [Libro en línea]. Salamanca, España: Ediciones Sígueme.Disponible:https://escuelacriticavaldiviana.files.wordpress.com/2012/06/levi nas-1961-totalidad-e-infinito_ocr.pdf [Consulta: 2015, marzo 20]

Morán, P. (1996). La instrumentación didáctica en la perspectiva de la didáctica critica en fundamentación de la didáctica. México: Gernika.

Peleteiro, I. (2006). Pedagogía Social Y Didáctica Crítica: Consideraciones para una práctica educativa orientada a los sectores en situación de desventaja y exclusión social. [Documento en línea]. R58-9.qxp. Disponible: http://cmap.upb.edu.co/rid=11 
96125300531_401020016_1037/Peleteiro\%20Pedagog\%C3\%ADa\%20social\%20y\%20did \%C3\%A1ctica\%20cr\%C3\%ADtica.pdf [Consulta: 2015, Abril 15]

Ranciere, J. (2003). El maestro ignorante. Barcelona, España: Laertes.

Rodríguez, M. (1997). Hacia una didáctica crítica. Madrid, España: La Muralla.

Savater, F. (1997). El valor de educar. Barcelona, España: Ariel.

Tunnermann, C. (2000). Universidad y sociedad. Balance histórico y perspectivas desde Latinoamérica. Comisión de Estudios de Postgrado. Facultad de Humanidades y Educación. Universidad Central de Venezuela. Caracas: Imprimatur.

Uzuriaga, L; Vivian, L; Martínez, A. (2006). Retos de la enseñanza de las matemáticas en el nuevo milenio. [Documento en línea]. Scientia ET. Technica, XII (31), 265-278 Disponible: http://www.redalyc.org/pdf/849/84911639046.pdf [Consulta: 2015, enero 29] 